\title{
CSA\&S/PV: Parallel Framework for Complex Systems Simulation
}

\author{
Ewa Niewiadomska-Szynkiewicz ${ }^{1,2}$ and Maciej Żmuda ${ }^{2}$ \\ 1 Research and Academic Computer Network (NASK), Wawozowa 18, \\ 02-796 Warsaw, Poland \\ e-n-s@ia.pw.edu.pl \\ http://www.nask.pl \\ 2 Institute of Control and Computation Engineering, Warsaw University of \\ Technology, Nowowiejska 15/19, 00-665 Warsaw, Poland \\ mzmuda@elka.pw.edu.pl \\ http://www.ia.pw.edu.pl
}

\begin{abstract}
The paper1 presents an integrated environment CSA\&S/PV (Complex Systems Analysis 85 Simulation - Parallel Version), which can be used for design and simulation of large scale systems such as data networks, complex control systems and many others operating in inherently parallel environments. CSA\&S/PV provides framework for simulation experiments carried out on parallel computers. It was used to investigate several real-life problems. Two examples are provided to illustrate the operation of the presented software tool: a hierarchical optimization algorithm for flow control in communication and computer networks and a hierarchical control structure for flood control in water networks.
\end{abstract}

\section{Introduction}

It is natural to model complex systems as a set of calculation processes, which can then be handled by distributed machines or processors. Recently parallel and distributed simulation has been an active research area, [34]. Distributed simulation allows to reduce the computation time of the simulation program, to execute large programs, which cannot be executed on single machine, to reflect better the structure of the physical system, which usually consists of several components.

In order to perform simulation experiments efficiently it is required to have good software tool. Numerous systems have been engineered to aid programmers. In most cases they are specified to certain solving problems 1871910. Since parallel and distributed simulation is fast becoming the dominant form of model execution the focus is on experiments carried on parallel and distributed hardware platforms. High Level Architecture (HLA) standard for distributed

\footnotetext{
${ }^{1}$ This work was supported by Research and Academic Computer Network (NASK) and Polish Committee for Scientific Research under grant 7 T11A 02220.
} 
discrete-event simulation was defined by the United States Department of Defense. During last years numerous integrated environments for parallel and distributed processing were developed. These software tools apply different techniques for synchronization and memory management, and focus on different aspects of distributed implementation. Many of them are built in Java, 5. SimJava was among the first publically released simulators written in Java. This paper deals with the description of an integrated framework for parallel simulation CSA\&S/PV Complex Systems Analysis \& Simulation - Parallel Version.

\section{Description of CSA\&S/PV}

CSA\&S/PV presented in preliminary sequential version, in [6], is the software environment for different types of real systems simulation. The main idea of its development was to minimize user's effort during design and simulation of complex systems. CSA\&S/PV provides a framework, which allows to perform simulations on parallel computers. It offers the graphical environment (shell) for supporting the considered case study implementation and a library of functions providing communication between the user's applications (LPs) and the system interface. CSA\&S/PV manages calculations and communication between running processes, provides tools for on-line monitoring of the computed results. The asynchronous version of simulation is applied, 44. In asynchronous simu-

\begin{tabular}{|l|l|l|l|l|}
\hline $\begin{array}{l}\text { receiver } \\
\text { address }\end{array}$ & lags & $\begin{array}{l}\text { time } \\
\text { stamp }\end{array}$ & $\begin{array}{l}\text { transmission } \\
\text { delay }\end{array}$ & data \\
\hline
\end{tabular}

Fig. 1. Contents of messages from LPs simulating the considered physical system graph)

lation shared data object, the global clock and global event list, are discarded. Each logical process maintains its own local clock (LVT - Local Virtual Time). The local time of different processes may advance asynchronously. Events arriving at the local input message queue of a logical process are executed according to the local clock and schedule scheme. LPs can operate in two modes:

time-driven: The increment of $L V T$ of each logical process is fixed and defined by the user. LP is executed every defined time step (repetition time), which means that $L V T$ changes at regular intervals. We assume that for different LPs different repetition times may be introduced.

event-driven: Logical processes are executed after event occurrence. LVTs change at irregular intervals. A conservative scheme similar to the CMB algorithm, described in [3] is used for synchronization. Each event is executed only if it is certain that no event with an earlier time-stamp can arrive. At current time $t$ each logical process LPi computes the minimum time $L V T_{i}=\min _{j \in N(i)}\left(t_{i j}+\tau_{i j}\right)$, where $t_{i j}$ is the time-stamp of the last message 
received from $\mathrm{LPj}$ process, $N(i)$ is a set of processes transmitting data to LPi and $\tau_{i j}$ is a transmission delay from node $j$ to $i$ (transfer cost). Next, every LPi simulates all events with time-stamps smaller than the $L V T_{i}$. The processes exchange messages as presented in Fig. 1. When the execution of the considered event begins, LPi sends to all their neighbours null messages with the time-stamp $L V T_{i}+\Delta T_{i}$ (where $\Delta T_{i}$ denotes the pending events time), which is the earliest possible time of next message. Null messages are used to announce absence of messages with new data.

Both types of LPs can be executed during one simulation.

\subsection{System Structure}

CSA\&S/PV is composed of five components (see Fig. 2): 1) shell - the Graphical User Interface (GUI), responsible for user-system interaction, 2) calculation module (manager) - the system kernel that manages calculations and communication between running processes, 3) communication library - the library of functions that provides communication between the graphical shell and the system kernel, 4) user library - the library of functions that provides interface between the user application and "manager" (system kernel), 5) user applications - simulators of the physical subsystems (developed by the user).

The user's task is to implement the subsystems' simulators corresponding to the nodes of the considered graph and responsible for adequate physical systems simulation. These modules may be written in Java or C, C++. As it was mentioned above the CSA\&S/PV package supplies the library of functions, which provides the interface between these programs and the system. This allows the user to focus on the numerical part of the program only. In addition if the functions unique to the operating system are not used by the user, applications can be moved as necessary between different computing platforms.

In general each user's application consists of six functions: csasInit, the task of which is to prepare the environment for future calculations and to calculate the initial conditions, csasExecuteArgs, which gathers data for calculations from CSA\&S/PV, csasExecute, which provides calculations (the main part of the user's application), csasExecuteResults, which sends the results of calculations to CSA\&S/PV, csasStore, the task of which is to store all current calculation results after system termination (simulation can be continued), csasEnd, an additional function for removing all data structures dynamically allocated during program operation.

Each node independently executes its program and communicates each other and with system kernel using user library functions. The system kernel manages only communication between the user interface (shell) and calculation processes corresponding to the nodes.

\subsection{Implementation Details}

The CSA\&S/PV system has been implemented in Java, so it may operate under MS-Windows, Windows-NT and Unix operating systems. All calculation 


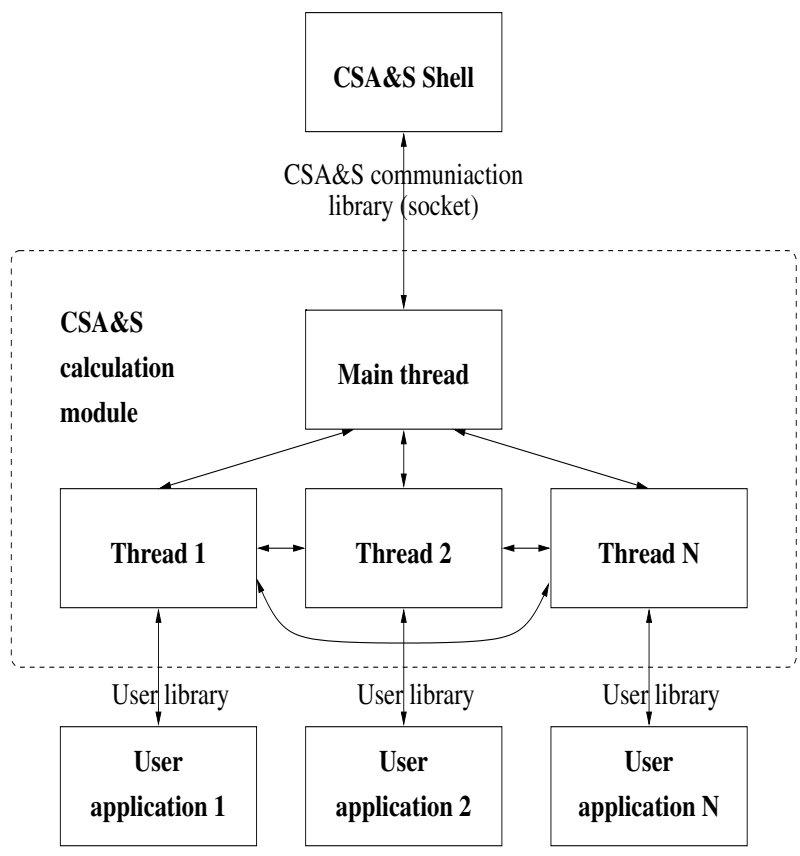

Fig. 2. The architecture of the CSA\&S/PV system

processes communicate with each other via shared memory. The mechanism for parallel implementation is based on threads (see Fig. 2). Each calculation process (user's application) runs a thread. Because the software system is heterogeneous (shell and manager may operate under different operating systems) CSA\&S/PV establishes a two-way socket connection between the user interface and the system kernel. The additional library of functions that provide communication between these two processes was developed.

\subsection{Simulation under CSA\&S/PV}

During simulation experiment performed under CSA\&S/PV one can distinguish two main stages: preparatory stage and experimental stage. At the preparatory stage the model and the properties of the system to be simulated are investigated. The calculation process is partitioned into several subsystems (subtasks) with respect to functionality and data requirements. The directed graph of the considered system $G=(N, L)$ is created and entered into CSA\&S/PV using graph editor. The set of nodes is equal $N$ with node $i$ representing the $i$-th subsystem $(i=1, \ldots, N)$. The set of links is equal $L$. The presence of an arc $(i, j) \in L$ indicates the possibility that the $i$-th subsystem influences the $j$-th subsystem. Each node of the graph represents the program (LP) executing the tasks of the simulated physical subsystem. As it was mentioned above all these 
programs have to be prepared by the user and they must be ready to run. As far as the CSA\&S/PV system is concerned, the goal of each node application program is to gather data from the connected nodes and to generate another data for the other nodes. Within the next step the user is asked to provide some information related to the nodes of the considered graph. The information includes: the name of the calculation program corresponding to each node, the repetition time period (if necessary), the decision delay - time required to execute the events in the physical application. Next the user is asked to provide some information related to all inputs of these nodes: the name of each input, the transmission delay related to data transmission to a particular location. The currently considered graph of simulated system may be saved into the disc file. In this way the application can be used in many future simulations.

The experimental stage begins when all decisions regarding the simulated system are made. The simulation time horizon is defined and the experiment starts. The adequate programs corresponding to the nodes of the system graph are executed. The results of the calculations are displayed. The user employs monitoring and analysis of the current situation. All results may be recorded into the disc file during the experiment. The simulation may be interrupted and restarted. There is a possibility to extend the simulation horizon if it is desired.

\section{Practical Examples}

The CSA\&S/PV software package was used to perform simulations of several large scale systems. In this paper the case studies connected with application of hierarchical technics to optimization and control are described. The results of analysis of pricing algorithms for data networks and systems for operational flood control in multiple reservoir system obtained under CSA\&S/PV are presented.

\subsection{Optimization Network Flow Control}

The first considered case study was related to the optimization approach to flow control in computer or communication networks. The asynchronous link algorithm for pricing of network services, based on the Price Method was implemented and tested. The detailed description of this method together with the discussion of its convergence one can find in [2].

Consider a network consisting of a set $L=\left\{1, \ldots, L_{n}\right\}$ of unidirectional links of capacities $c_{l}, l \in L$ and a set $S=\left\{1, \ldots, S_{m}\right\}$ of traffic sources. Each source is defined by a fourtuple $\left(L(s), U_{s}\left(x_{s}\right), x_{s_{\min }}, x_{s_{\max }}\right)$, where $x_{s}$ denotes transmission rate, $U_{s}\left(x_{s}\right)$ source utility function defined over interval $X_{s}=\left[x_{s_{\text {min }}}, x_{s_{\max }}\right] \subseteq R_{+}, x_{s_{\min }}, x_{s_{\max }}$ respectively, minimum and maximum transmission rates. For each link $l$ let $S(l)$ be the set of sources that use the link $l$, so $l \in L(s)$ if and only if $s \in S(l)$. The objective is to maximize the aggregate source utility over their transmission rates, so the flow optimization problem can be formulated as follows:

$$
\max _{x_{s} \in X_{s}} \sum_{s} U_{s}\left(x_{s}\right), \quad \sum_{s \in S(l)} x_{s} \leq c_{l}, \quad l \in L_{n}
$$


If the feasible set is nonempty and the performance function is strictly concave then the unique maximizing solution, $\hat{x}$ exists (see [2]).

IP networks consist of many subsystems, i.e. sources, routers, etc. They are constrained by the common resources - network capacity. The usage of methods with decomposition and coordination seems to be natural for such systems control. The optimization problem (11) can be solved by the Price Method (dual method using price coordination) in the parallel or distributed environment.

Define the Lagrange function of (11)

$$
\begin{aligned}
L(x, \lambda) & =\sum_{s} U_{s}\left(x_{s}\right)-\sum_{l} \lambda_{l}\left(\sum_{s \in S(l)} x_{s}-c_{l}\right)= \\
& =\sum_{s}\left(U_{s}\left(x_{s}\right)-x_{s} \sum_{l \in L(s)} \lambda_{l}\right)+\sum_{l} \lambda_{l} c_{l}
\end{aligned}
$$

where $\lambda_{l} \geq 0$, i.e. the Lagrange multipliers associated with capacity constraints denote the link prices.

We can formulate the local (source) and coordinator level optimization problems:

LPs : $s=1, \ldots, S_{m}$, for given $\lambda_{l}$ find maximum w.r.t. $x_{s}$ of the local performance index

$$
\max _{x_{s} \in X_{s}}\left[L_{s}\left(x_{s}, \lambda\right)=U_{s}\left(x_{s}\right)-x_{s} \sum_{l \in L(s)} \lambda_{l}\right]
$$

CP : for the results of LPs find minimum w.r.t. $\lambda_{l}$ of the coordinator performance index

$$
\min _{\lambda_{l} \geq 0, l=1, \ldots, L_{n}}\left[\varphi(\lambda)=\sum_{s} L_{s}\left(\hat{x}_{s}, \lambda^{s}\right)+\sum_{l} \lambda_{l} c_{l}\right]
$$

where $\lambda^{s}=\sum_{l \in L(s)} \lambda_{l}$.

Synchronous and asynchronous distributed algorithms for prices computing were proposed by Low and Lapsley in [2. They are the descent algorithms for the dual function minimization, with the price projection on $R_{+}^{S_{m}}$. In the synchronous version the $l$-th link price at the iteration instant $k+1$ is calculated as follows:

$$
\lambda_{l}(k+1)=\left[\lambda_{l}(k)-\gamma \frac{\partial \varphi(\lambda(k))}{\partial \lambda_{l}}\right]_{+}=\left[\lambda_{l}(k)+\gamma\left(\sum_{s \in S(l)} \hat{x}_{s}(k)-c_{l}\right)\right]_{+}
$$

where $[y]_{+}=\max (y, 0)$ and $\gamma$ is sufficiently small step size.

Thus, in the (5) approach all sources receive, at a given time instant $k$, prices $\lambda_{l}(k)$, compute respective source prices $\lambda^{s}(k)$ and calculate optimal source rates $\hat{x}_{s}(k)$ solving LPs problems. The obtained values of the source rates $\hat{x}_{s}(k)$ are then send to the links and the new link prices $\lambda_{l}(k+1)$ are computed according to $(5)$.

In the case of asynchronous approach both sources and link algorithms use weighted averages of the past values of the link prices and the locally optimal source rates. So, the $l$-th link price at the iteration instant $k+1$ is 


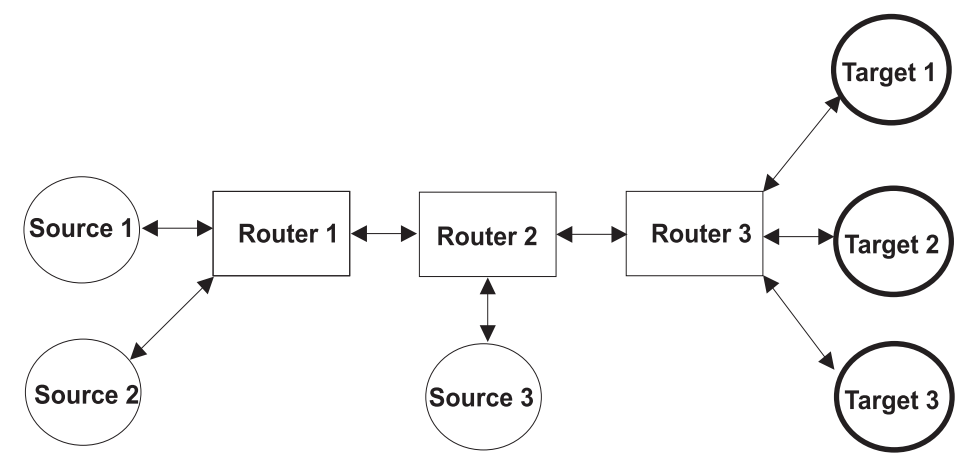

Fig. 3. Considered IP network under CSA\&S/PV system

calculated according to (5) assuming $\hat{x}_{s}(k)=\sum_{k^{\prime}=k-k_{0}}^{k} a_{l s}\left(k^{\prime}, k\right) x_{s}\left(k^{\prime}\right)$ with $\sum_{k^{\prime}=k-k_{0}}^{k} a_{l s}\left(k^{\prime}, k\right)=1$, for all $k, l$ and $s \in S(l) ; k_{0}$ denotes the length of past window taken into account. Respectively, the $s$-th source rate at time $k+1$ is calculated solving LPs, assuming $\lambda^{s}(k)=\sum_{l \in L(s)} \sum_{k^{\prime}=k-k_{0}}^{k} b_{l s}\left(k^{\prime}, k\right) \lambda_{l}\left(k^{\prime}\right)$ with $\sum_{k^{\prime}=k-k_{0}}^{k} b_{l s}\left(k^{\prime}, k\right)=1$, for all $k, s$ and $l \in L(s)$.

The described above asynchronous pricing algorithm was applied for flow control in experimental computer network, as presented in Fig. 3. It consists of nine nodes: three sources, three routers, three destination nodes and eight bidirectional links (the algorithm requires communication between sources and routers). The maximal capacity of the links Router1-Router2 and Router2-Router3 was equal 290. The capacity of other links was unlimited. The network was implemented in CSA\&S/PV system using nine calculation processes. The processes exchanged messages as presented in Fig. 1, containing adequate data: link prices - messages from routers and source rates - messages from sources. All calculation processes corresponding to the nodes in Fig. 3 could communicate and update their controls asynchronously at different time instants, with different frequencies, and after transmission delays. The utility functions $U_{s}$ of the sources were set to $\alpha_{s} \log \left(1+x_{s}\right)$, with $\alpha_{s}=10^{4}$ for all sources. Only the last received rate $x_{s}(\tau)$, for $\tau \in k-k_{0}, \ldots, k$ was used to estimate the locally optimal source rates and the link prices, i.e. $a_{l s}$ and $a_{l s}$ were set to 1 for $k^{\prime}=k$ and 0 for $k^{\prime}<k$. Each source transmitted data for a total of 120000 time units; source 1 started transmission at time 0 , source 2 at time 40000, source 3 at time 80000 . The whole horizon of simulation was equal 240000 time units. The goal was to test the convergence of the algorithm w.r.t. value of the step size in (5) and transmission delays in the network. Several experiments were performed taking into account different values of step size $\gamma=\{1 e-3,1 e-4,1 e-5\}$, and different transmission delays $\tau_{D}=\{1,10,100\}$ time units. It was assumed that the transmission was delayed for all links. The results are presented in table 1 and figures 4, 5. Table 1 contains percentage of rejected data packets w.r.t. all packets passed during the experiment. Figures 4 and 5 show respectively the destination receive rates 
Table 1. Percentage of rejected packages with respect to different values of $\gamma$ and $\tau_{D}$

\begin{tabular}{|l|l|l|l|}
\hline & $\tau_{D}=1$ & $\tau_{D}=10$ & $\tau_{D}=100$ \\
\hline$\gamma=1 \mathrm{e}-3$ & 14.59 & 15.62 & 98.41 \\
\hline$\gamma=1 \mathrm{e}-4$ & 63.54 & 63.55 & 65.51 \\
\hline$\gamma=1 \mathrm{e}-5$ & 92.22 & 92.23 & 92.28 \\
\hline
\end{tabular}

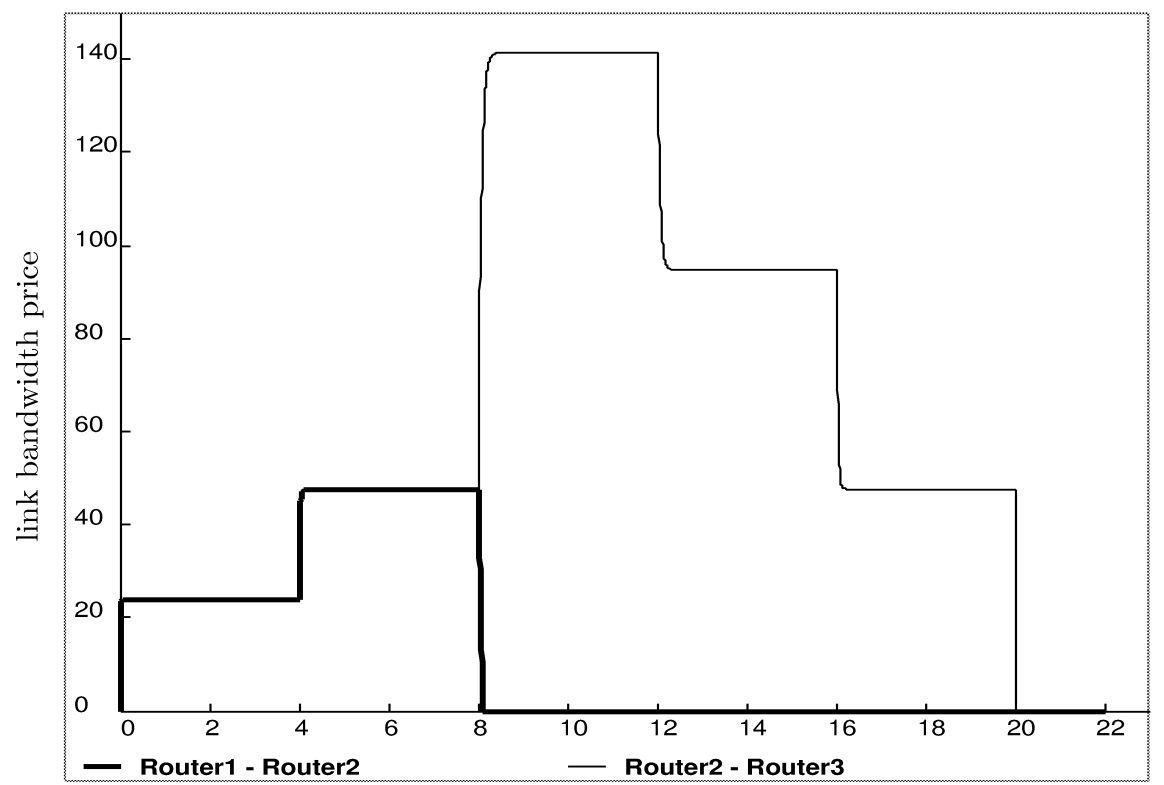

time $[10000 \times$ time unit $]$

Fig. 4. The link prices for $\gamma=1 e-3$ and $\tau_{D}=1$

and the link prices. We can observe that the source rates adjusted dynamically as new sources started or stopped transmitting. As expected, the number of rejected packages increased for the longer transmission delay. For $\tau_{D}=100$ and step size $\gamma=1 e-3$ the algorithm was not convergent to the global optimum (see Table 1). After decreasing the step size better solution was achieved. On the other hand the decreasing $\gamma$ took longer for the algorithm (15) to arrive at the proper price values. In the case of very small value $(\gamma=1 e-5)$ the algorithm seemed to track the optimum but the solution was not reached. The presented results show that the considered pricing algorithm for flow control is very sensitive to the value of the step size in optimization proces. The estimation of the proper $\gamma$ may involve many problems especially in the case of huge network traffic. 


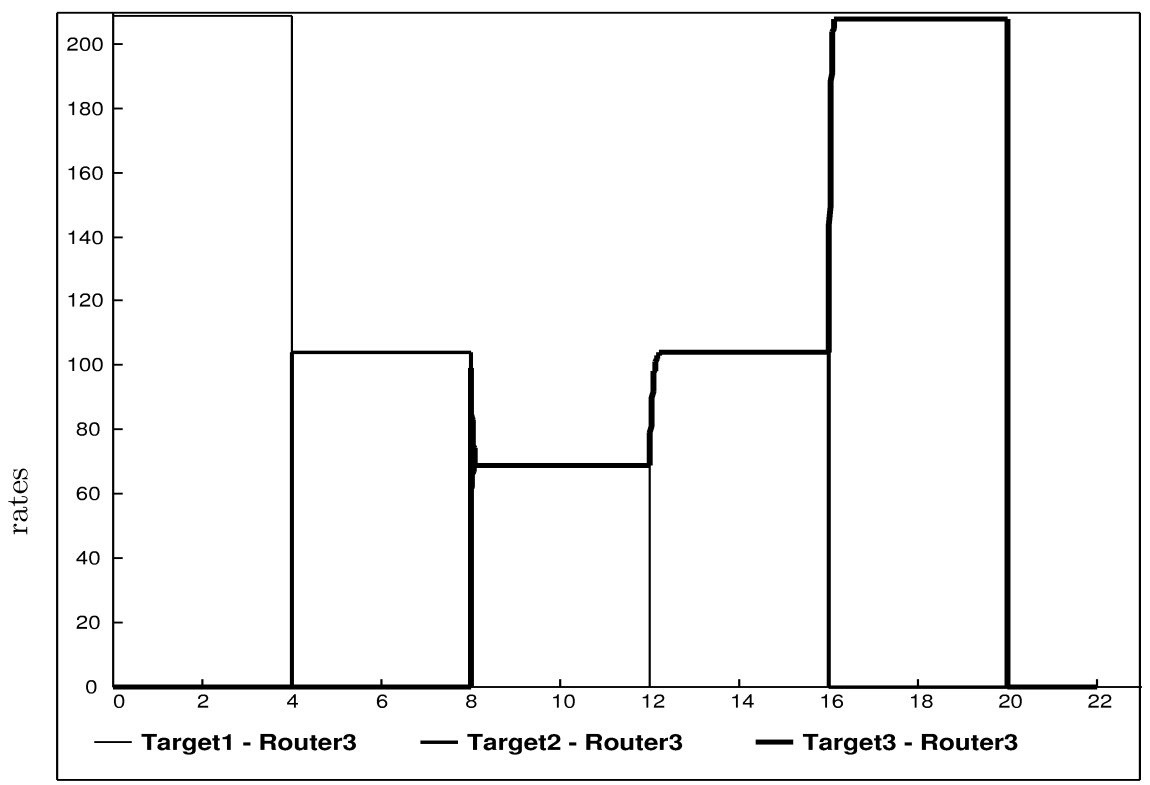

time $[10000 \times$ time unit $]$

Fig. 5. The source rates for $\gamma=1 e-3$ and $\tau_{D}=1$

\subsection{Flood Control in Multireservoir System}

The second case study, implemented under CSA\&S/PV was related to flood control in the Upper Vistula river-basin system in the Southern part of Poland. Three retention reservoirs, located on Sola, Raba, Dunajec rivers were considered. The optimal release problem was defined as the problem of minimization the flood damages related to the peak flows at the measurement points in the whole river system. The hierarchical control mechanism (HDM) for reservoirs management, capable of satisfying the global objectives was investigated (see Fig. 6). This mechanism is based on the use of the repetitive optimization of the outflow trajectories, using the predicted inflows - forecasts (see [7] for details). It incorporates two decision levels each (see Fig. 6): the upper level with the control center (coordinator) and the local level formed by the operators of the reservoirs. The local decision rules are designed in such way that a central authority, the coordinator, may adjust them in the process of periodic coordination so as to achieve the coordination of reservoirs in minimizing the global damages. Hence, the decision problem of the $i$-th local reservoir operator $(i=1,2,3)$ at time $t_{l}$ is as follows:

$$
\min _{u_{i}}\left[q_{i}\left(u_{i}(.), a_{i}\right)=\max _{t \in\left[t_{l}, t_{f}\right]}\left(u_{i}(t) \cdot \alpha_{i}(t)\right)\right]
$$

where $\left[t_{l}, t_{f}\right]$ denotes local level optimization horizon, $q_{i}$ local cost function, $a_{i}$ parameters specified by the coordinator. The vector $a_{i}$ of coordinating param- 


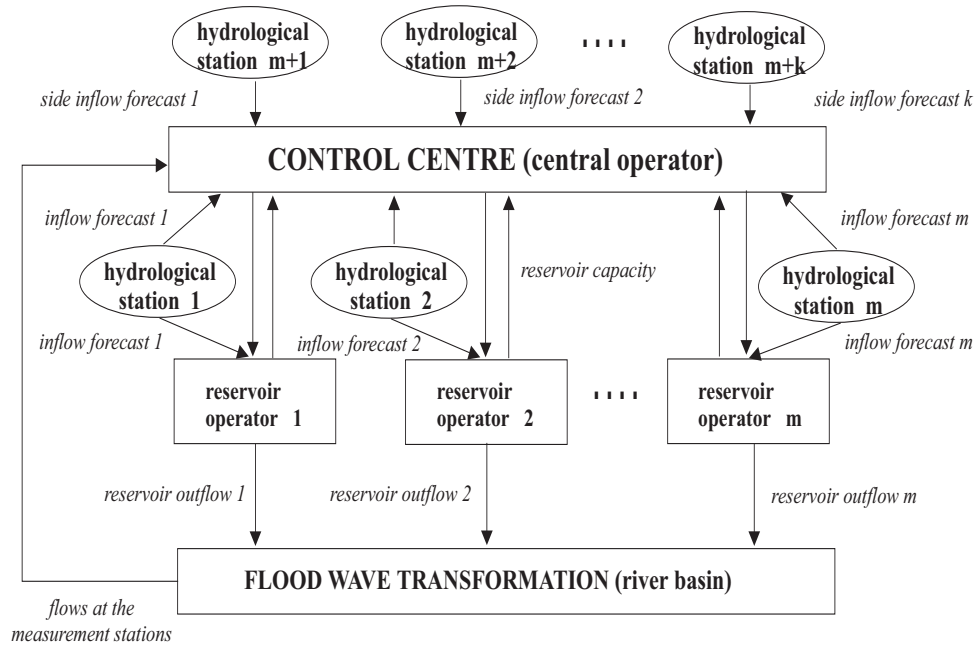

Fig. 6. Flood control in Vistula reservoir system

eters for the $i$-th reservoir is related to the weighting function $\alpha_{i}($.$) defined as$ follows: $\alpha_{i}(t)=1+\left(c_{i}-1\right) \cdot 1\left(t-T_{i}^{\star}\right)$, i.e., $\alpha_{i}(t)=1$ for $t \in\left[t_{l}, T_{i}^{\star}\right)$ and $\alpha_{i}(t)=c$ for $t \in\left[T_{i}^{\star}, t_{f}\right]$.

The goal of the control center is to calculate the optimal values of parameters $a$, such that minimize the damages in the whole river basin.

$$
\min _{a \in A} J\left(Q_{\left[t_{c}, t_{f}\right]}\right), \quad Q(t)=F\left(Q\left(t_{c}\right), \hat{u}_{\left[t_{c}, t\right]}(a), \bar{d}_{\left[t_{c}, t\right]}^{t_{c}}\right)
$$

where $\left[t_{c}, t_{f}\right]$ denotes control center optimization horizon $\left(t_{c} \leq t_{l}\right), Q(t)$ vector of flows at the measurement points, $Q\left(t_{c}\right)$ vector of real flows measured at time $t_{c}, \bar{d}^{t_{c}}$ vector of forecasts of all the inflows calculated at time $t_{c}, \hat{u}$ vector of optimal outflows from the reservoirs (associated with vector of parameters $a), J\left(Q_{\left[t_{c}, t_{f}\right]}\right)$ a performance (loss) function. Every iteration of the optimization process the value of $J($.$) is computed based on simulation of the lower decision$ level (reservoirs' operators) and flow transformation in the whole river basin.

Presented control structure was implemented under CSA\&S/PV. The whole system was decomposed into several subsystems (processes) associated with the nodes in Fig. 6] control center, (coordination parameters calculation), reservoirs' operators (releases calculation), hydrological stations (inflow forecasts computing), rivers (flow transformation). Simulations were performed for the set of historical data. The results obtained for presented control system were compared with an independent reservoir management. The robustness of hierarchical control mechanism with respect to decision and transmission delays was tested too. 


\section{Conclusion}

Simulation tools play an important role in the computer-aided analysis and design of complex control systems. CSA\&S/PV is such a tool written in Java programming language on different platforms. The presented applications demonstrate the effectiveness and efficiency of the CSA\&S/PV system. In both case studies CSA\&S/PV was used as an environment for testing hierarchical techniques for control and optimization. It should be pointed that the considered applications could be solved in sequential manner but in both cases parallel computations were natural. In general the CSA\&S/PV framework is suitable to solve many other small and large scale problems based on computer analysis and simulation. The package may be easily extended by "toolbox" of software modules, which are specific to a chosen case study.

\section{References}

1. Di, Z., Mouftah, H.T., QUIPS-II: a Simulation Tool for the Design and Performance Evaluation of Diffserv-based Networks, Computer Communications, 25, pp.11251131, 2002.

2. Low, S., Lapsey, D.E., Optimization Flow Control I: Basic Algorithm and Convergence, IEEE/ACM Transactions on Networking, 7(6), 1999.

3. Misra J., Distributed Discrete-Event Simulation, Computing Surveys, Vol. 18, No. $1,1986$.

4. Nicol D.M., Fujimoto R., Parllel Simulation Today, Annals of Opertions Research, Vol. 53, pp. 249-285, 1994

5. Nicol D.M., Johnson, M., Yoshimura, A., Goldsby, M., Performance Modeling of the IDES Framework, Proc. of the Workshop on Parallel and Distributed Simulation, pp.38-45, Lockenhaus, Austria, 1997.

6. Niewiadomska-Szynkiewicz E., Pooenik P., Bolek P., Malinowski K., Software Environment for Complex Systems Analysis and Simulation, Preprints IFAC/IFORS/IMACS Symposium "Large Scale Systems: Theory and Applications", London, UK, 1995

7. Niewiadomska-Szynkiewicz, E., Software Environment for Simulation of Flood Control in Multiple-Reservoir Systems, Proc. International Conference "Advances in Hydro-Science and Engineering" ICHE2002, Warsaw, Poland, 2002.

8. ns-2 (network simulator). http://www.isi.edu/nsnam/ns/ns-documentation.html.

9. Omnet (network simulator). http://www.hit.bme.hu/phd/vargaa/omnetpp.htm.

10. Szymañski, B., Liu, Y., Sastry, A., Madnani, K., Real-Time On-Line Network Simulation, Proc. 5th IEEE International Workshop on Distributed Simulation and Real-Time Applications DS-RT 2001, pp.22-29, Los Alamitos, CA, 2001. 